\title{
Chronic obstructive pulmonary disease in the emergency department
}

\section{La maladie pulmonaire obstructive chronique à l'urgence}

I In the present issue of the Canadian Respiratory Journal, Rowe et al (1) compare emergency room (ER) experiences of chronic obstructive pulmonary disease (COPD) patients from Canada with those of COPD patients from the United States (US). The study was a large multicentre effort that enlisted 29 emergency departments five in Canada - and essentially reviewed consecutive patients entering the ERs for two weeks in 2001. The patients were interviewed, and permission to use their data was acquired. Patient charts were reviewed and a follow-up telephone call was made two weeks after the initial event. A largely successful effort was made to establish the validity of the COPD diagnosis in a subset of patients. No effort was made to influence patient management, so the result is a descriptive study. Demographics and pre-event status, including symptoms, medications and comorbidities, were assessed, as were presenting symptoms and assessments, treatments and outcomes. The study represents a major effort on the part of the investigators.

Overall, there were few differences between Canadian and American results. The obvious difference in third-party payers was noted, as was the fact that Canadians were somewhat more likely to have a primary care physician, had longer stays in the ER (perhaps due to bed shortages), and were more likely to receive antibiotics both in the ER and on discharge. The first of these differences is, of course, intrinsic to the differing health care systems in Canada and the US. Other differences are hard to evaluate, because it is not clear whether the Canadian and American ERs served similar populations; the American sample was biased toward inner-city academic centres because they were more likely to serve the disadvantaged, and this was probably less so in Canada. As the authors state, participating ERs were self-selected, and therefore not generally representative of ERs in either country.

Similarities between countries in terms of the type of patient seen, the treatment received in the ER and the outcomes of the visit were much more striking than the differences, which were at times puzzling and not reassuring. Although approximately two-thirds of the patients had had a previous hospital admission for COPD, only one-third complained of symptoms "most of the time" before study entry; these results seem to contradict each other, unless the patients were not very sick, which seems unlikely. Most, but not all, of the patients were using bronchodilators before entry, but only one-half were on inhaled corticosteroids, undertreated by current standards (2). Interestingly, Canadian patients were more symptomatic on ER entry, but the significance of this is hard to evaluate. Arterial oxygen saturation was measured by oximetry in approximately $70 \%$ of the patients, but fewer than $30 \%$ ans le présent numéro de la Revue canadienne Ue pneumologie, Rowe et coll (1) ont comparé les expériences des patients atteints d'une maladie pulmonaire obstructive chronique (MPOC) du Canada à ceux des États-Unis (ÉU). L'étude, un grand effort multicentrique dans 29 départements d'urgence, dont cinq du Canada, a consisté à analyser les patients consécutifs qui ont fréquenté l'urgence pendant deux semaines en 2001. Les patients ont passé une entrevue et les auteurs ont obtenu l'autorisation d'utiliser leurs données. Ils ont révisé le dossier des patients et procédé à un suivi téléphonique deux semaines après l'événement initial. Ils ont fait un effort largement réussi en vue de valider le diagnostic de MPOC dans un sous-groupe de patients. Ils n'ont pas pris de mesures pour influer sur la prise en charge des patients. Les résultats sont donc descriptifs. Les auteurs ont évalué le statut démographique et avant l'événement, y compris les symptômes, les médicaments et les comorbidités, de même que les symptômes et les évaluations à la consultation, les traitements et les issues. Cette étude représente un effort majeur de la part des chercheurs.

Dans l'ensemble, il y avait peu de différences entre les résultats canadiens et américains. La différence évidente des tiers payeurs a été soulignée, de même que le fait que les Canadiens étaient quelque peu plus susceptibles de disposer d'un médecin traitant, qu'ils demeuraient plus longtemps à l'urgence (peutêtre à cause de la pénurie de lits) et étaient plus susceptibles de recevoir des antibiotiques à la fois à l'urgence et à leur congé. Bien sûr, la première de ces différences provient des systèmes de santé différents au Canada et aux ÉU. Les autres différences sont difficiles à évaluer, parce qu'on ne sait pas si les urgences canadiennes et américaines servent des populations similaires. L'échantillon américain était biaisé vers les centres universitaires des quartiers pauvres parce qu'ils étaient plus susceptibles de soigner les populations défavorisées, ce qui était probablement moins le cas au Canada. Comme l'ont déclaré les auteurs, les urgences participantes se sont elles-mêmes proposées et n'étaient donc pas représentatives de l'ensemble des urgences des deux pays.

Les similarités entre les pays pour ce qui est du type des patients vus, du traitement reçu à l'urgence et des issues de la visite étaient beaucoup plus frappantes que les différences, qui étaient parfois curieuses et peu rassurantes. Même si environ les deux tiers des patients avaient déjà été hospitalisés à cause d'une MPOC, seul le tiers se sont plaints de symptômes « la plupart du temps » avant l'inscription à l'étude. Ces résultats semblent contradictoires, à moins que les patients n'aient pas été très malades, ce qui semble peu probable. La plupart des patients, mais pas tous, utilisaient un bronchodilatateur avant 
underwent measurement of arterial blood gases or expiratory flows; this seems low but also could indicate that the patients were not critically ill. ER treatments were as expected: inhaled bronchodilators were administered in virtually all patients and systemic steroids in approximately two-thirds; as noted above, antibiotics were more frequently used in Canada than in the US.

Outcomes were similar between countries and were not encouraging. Close to two-thirds of the patients seen were admitted to hospital, appearing to contradict the suspicion that they were not, on the whole, very sick. Furthermore, in response to the follow-up call at two weeks, the vast majority of patients had either relapsed or were no better. If this was true, and representative of the state of the art, it is very sobering. One could argue that the patients did not enjoy much benefit from the ER experience.

One could also argue that the study by Rowe et al (1) is outdated in that it describes the state of the art in 2001. While improvements in drug therapy since that time have not been dramatic, recent emphasis on self-management plans and education for patients with COPD (3) may well have decreased reliance on ERs as a source of care for COPD exacerbations. One hopes that this is the case.

Nick R Anthonisen MD Editor-in-Chief, Canadian Respiratory Journal

\section{REFERENCES}

1. Rowe BH, Cydulka RK, Tsai C-L, Clark S, Sinclair D, Carmango CA Jr. Comparison of Canadian versus American emergency department visits for chronic obstructive pulmonary disease exacerbation. Can Respir J 2008;6:302-310.

2. O'Donnell DE, Aaron S, Bourbeau J, et al. Canadian Thoracic Society recommendations for management of chronic obstructive pulmonary disease - 2007 update. Can Respir J 2007;14(Suppl B):5B-32B

3. Turnock AC, Walters EH, Walters JA, Wood-Baker R. Action plans for chronic obstructive pulmonary disease. Cochrane Database Syst Rev 2005:CD005074. leur inscription à l'étude, mais seulement la moitié prenaient des corticoïdes en aérosol; ils étaient donc sous-traités selon les normes actuelles (2). Fait intéressant, les patients canadiens étaient plus symptomatiques à leur arrivée à l'urgence, mais il est difficile d'en évaluer la signification. La saturation de l'oxygène artériel était mesurée par oxymétrie chez environ $70 \%$ des patients, mais moins de $30 \%$ avaient subi une gazométrie artérielle ou une débitmétrie expiratoire. Ces résultats semblent faibles, mais pourraient indiquer que les patients n'étaient pas gravement malades. Les traitements à l'urgence se passaient comme prévu : des bronchodilatateurs en aérosol étaient administrés à presque tous les patients et des stéroïdes systématiques, à environ les deux tiers d'entre eux. Comme on l'a déjà indiqué, les antibiotiques étaient plus utilisés au Canada qu'aux ÉU.

Les issues étaient similaires entre les pays et n'étaient pas encourageantes. Près des deux tiers des patients vus étaient hospitalisés, ce qui semble contredire la présomption que dans l'ensemble, ils n'étaient pas très malades. De plus, à l'appel de suivi au bout de deux semaines, la majorité des patients avaient rechuté ou n'étaient pas mieux. Si ce constat est vrai et représentatif du savoir-faire, il porte vraiment à réflexion. On pourrait avancer que les patients n'ont pas tiré grand profit de leur expérience à l'urgence.

On pourrait aussi avancer que l'étude de Rowe et coll (1) est dépassée puisqu'elle fait état du savoir-faire de 2001. Les améliorations de la pharmacothérapie n'ont pas tellement progressé depuis, mais l'accent mis récemment sur les plans d'auto-prise en charge et sur l'éducation des patients atteints de MPOC (3) pourrait bien avoir réduit la confiance envers l'urgence comme source de soins des exacerbations de MPOC. On espère que c'est le cas.

Nick R Anthonisen MD Rédacteur en chef, Revue canadienne de pneumologie

\section{RÉFÉRENCES}

1. Rowe BH, Cydulka RK, Tsai C-L, Clark S, Sinclair D, Carmango CA Jr. Comparison of Canadian versus American emergency department visits for chronic obstructive pulmonary disease exacerbation. Can Respir J 2008;6:302-310.

2. O'Donnell DE, Aaron S, Bourbeau J et coll. Canadian Thoracic Society recommendations for management of chronic obstructive pulmonary disease - 2007 update. Can Respir J 2007;14(Suppl B):5B-32B.

3. Turnock AC, Walters EH, Walters JA, Wood-Baker R. Action plans for chronic obstructive pulmonary disease. Cochrane Database Syst Rev 2005:CD005074. 


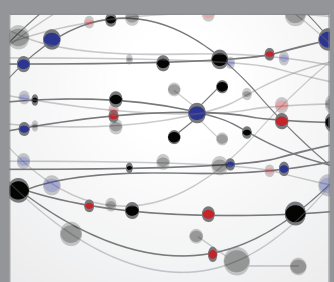

The Scientific World Journal
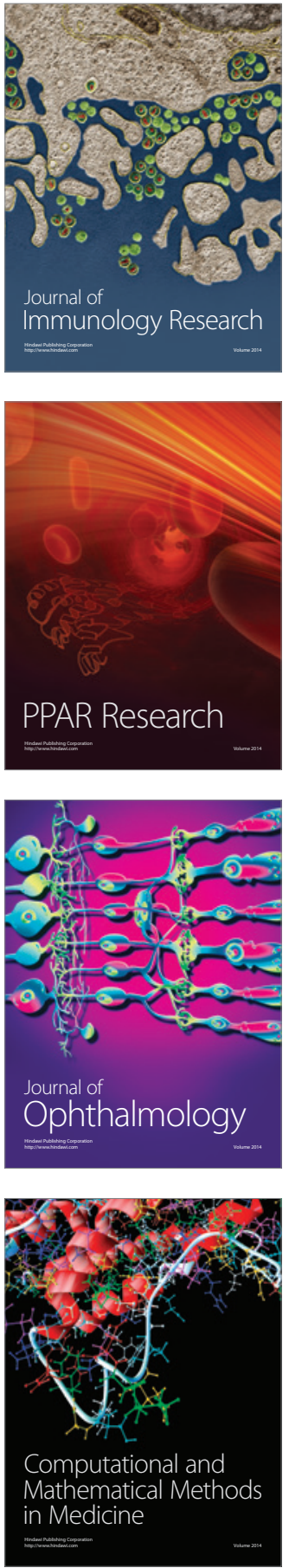

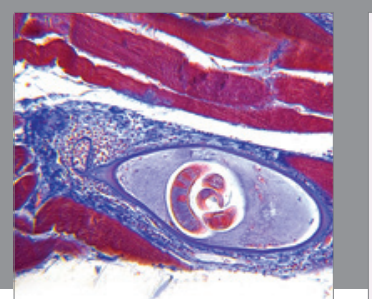

Gastroenterology Research and Practice

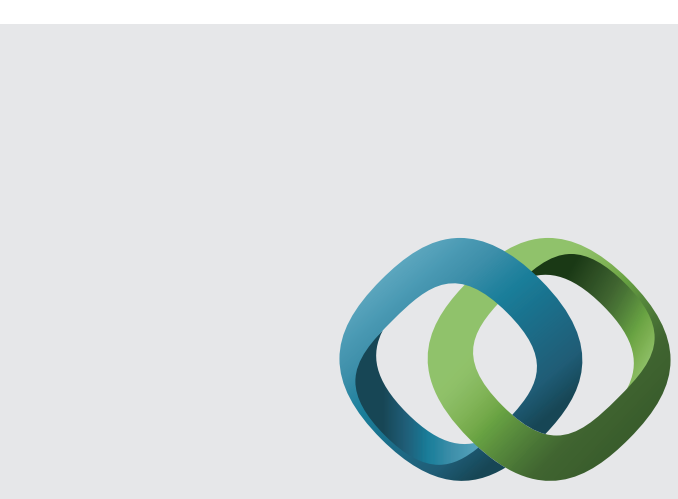

\section{Hindawi}

Submit your manuscripts at

http://www.hindawi.com
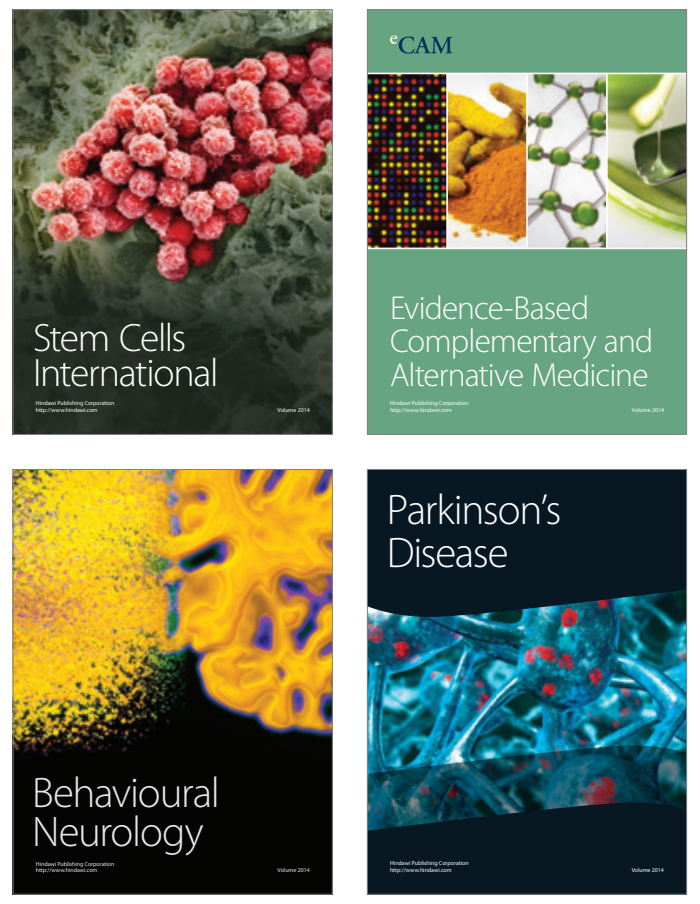
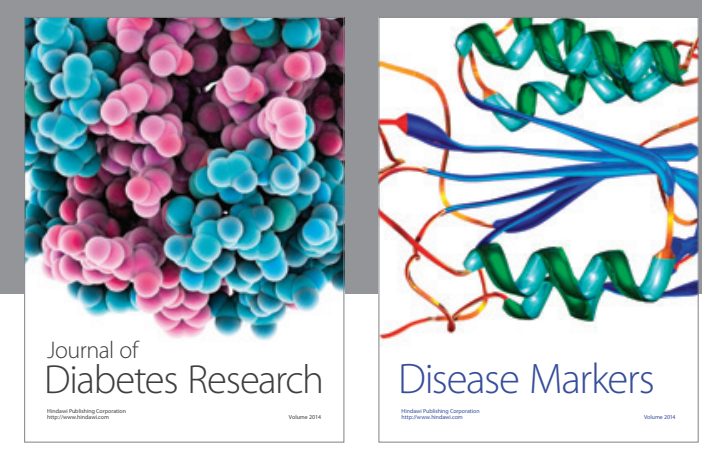

Disease Markers
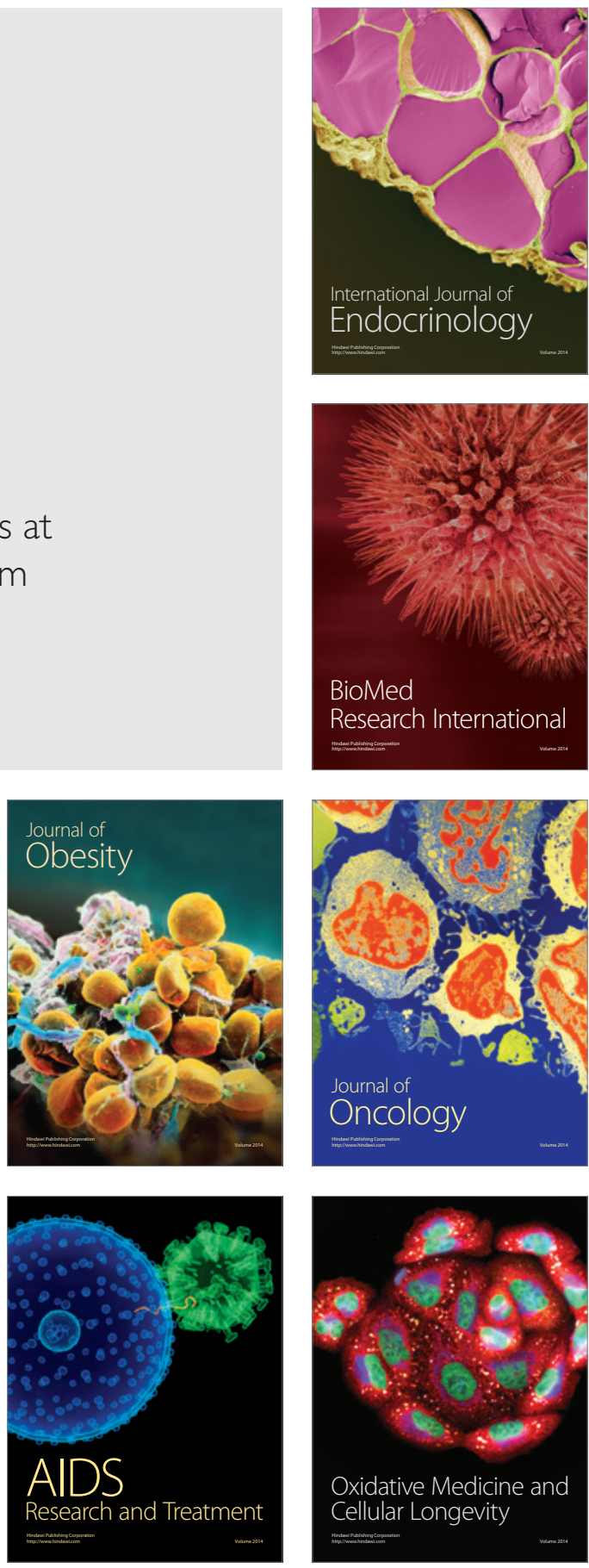